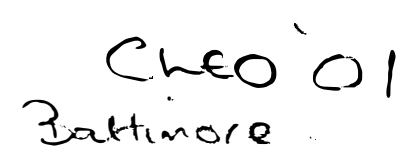

\title{
Femtosecond and picosecond fiber-feedback OPOs
}

\author{
T. Südmeyer, J. Aus der Au, R. Paschotta, and U. Keller \\ Ultrafast Laser Physics, Institute of Quantum Electronics \\ Swiss Federal Institute of Technology, ETH Hönggerberg - HPT, CH-8093 Zürich, Switzerland
}

\author{
P. G. R. Smith, D. J. Richardson, G. W. Ross, and D. C. Hanna \\ Optoelectronics Research Centre, University of Southampton \\ Southampton SO17 1BJ, United Kingdom
}

\begin{abstract}
We demonstrate a novel type of synchronously pumped high-gain optical parametric oscillator with feedback through a single-mode fiber. We present two fiber-feedback OPO systems, generating multi-watt average signal powers tunable around $1.5 \mu \mathrm{m}$ in 10 -ps and 800 -fs pulses.
\end{abstract}

\section{Introduction}

Synchronously pumped optical parametric oscillators (OPOs) are attractive sources of broadly wavelength-tunable ultrashort pulses as required for many applications, i.e. RGB display systems. We present a novel type of synchronously pumped OPO, which is based on feedback through a single-mode fiber in combination with a very high parametric gain and strong output coupling. This concept leads to compact, stable and powerful systems in the femtosecond and picosecond regime. We present a picosecond fiber-feedback OPO generating up to $4.4 \mathrm{~W}$ of average signal power in $\approx 10$-ps pulses tunable from $1485-1582 \mathrm{~nm}$. We also demonstrate a femtosecond fiberfeedback OPO that generates up to $2.7 \mathrm{~W}$ average signal power tunable from $1429-1473 \mathrm{~nm}$ in $700-900 \mathrm{fs}$ pulses. In contrast to many other OPOs in this pulse duration regime, this system is very insensitive against drifts of the OPO cavity length and does not require active stabilization of the cavity length. Because of the high parametric gain, fiber-feedback OPOs are unusually insensitive against intracavity losses. Even at low repetition rates, the setup is very compact since most of the resonator feedback path consists of the fiber.

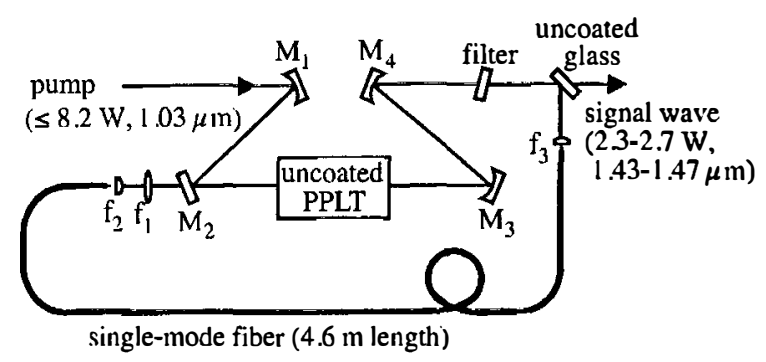

Fig. 1: Setup of the femtosecond fiber-feedback OPO ring cavity. $M_{1}-M_{4}=$ mirors, $f_{1}-$ $f_{3}$ : lenses, PPLT $=$ crystal of periodically poled $\mathrm{LiTaO}_{3}$.

\section{Femtosecond fiber-feedback OPO}

The femtosecond fiber-feedback OPO (Fig. 1) is based on a $22 \mathrm{~mm}$ long uncoated periodically poled $\mathrm{LiTaO}_{3}$ (PPLT) crystal, pumped with up to $8.2 \mathrm{~W}$ at $1030 \mathrm{~nm}$ from a passively mode-locked thin disk Yb:YAG laser [1] $\left(600^{\circ} \mathrm{fs}\right.$ pulse duration, $35 \mathrm{MHz}$ repetition rate). The crystal is operated at $\approx 150^{\circ} \mathrm{C}$ to avoid photorefractive damage 
and has different grating periods $(28.3 \mu \mathrm{m}-29 \mu \mathrm{m})$. After the crystal, $82 \%$ of the signal wave are coupled out, and part of the rest is launched into a $4.6 \mathrm{~m}$ long standard telecom fiber and fed back into the crystal.

We obtain up to $2.7 \mathrm{~W}$ of average signal power in the range $1429-1473 \mathrm{~nm}$ for a pump power of $8.2 \mathrm{~W}$ incident on the crystal (Fig. 1). We would expect to obtain even higher signal output powers in the order of $4 \mathrm{~W}$ by reducing the losses of several non-optimized optical components (uncoated PPLT, non-ideal output coupler). The pulse duration (FWHM) is typically around 700-900 fs with a spectral width of 3-4 nm (FWHM).

The adjustment of the cavity length is very uncritical: it can be varied over $0.5 \mathrm{~mm}$ (corresponding to more than one pulse width) with the output power reduced only by $6 \%$ (Fig. 3). No active cavity length stabilization is required. The performance of the system is unusually insensitive to intracavity losses: the output power is reduced by only $6 \%$ when a $10-\mathrm{dB}$ attenuator is inserted into the cavity. The operation is stable over hours.

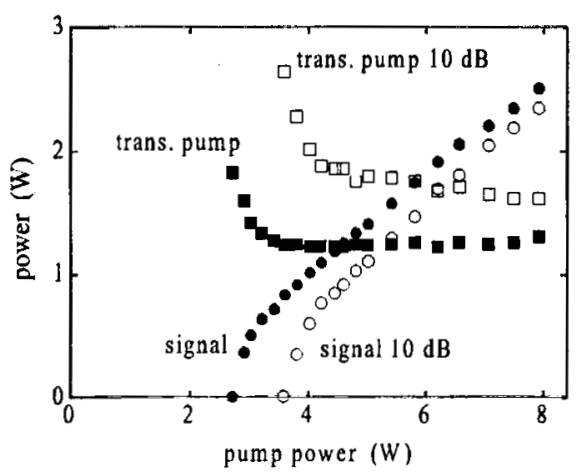

Fig. 2: Signal power (filled circles) and transmitted pump (filled rectangles) versus pump power for a signal wave of $1429 \mathrm{~nm}$ (grating period $28.3 \mu \mathrm{m}$ ). Open circles and rectangles: same with a $10-\mathrm{dB}$ attenuator in the feedback loop.

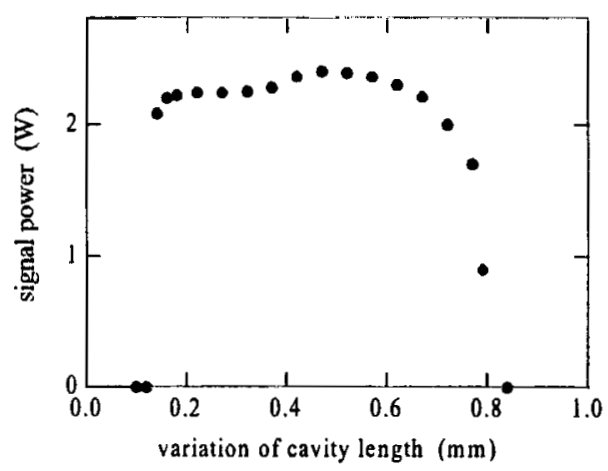

Fig. 3: Variation of signal output power with cavity length.

\section{Picosecond fiber-feedback OPO}

Very recently, we constructed a similar $\mathrm{OPO}$ as described above, but using a $19 \mathrm{~mm}$ long multi-grating periodically poled $\mathrm{LiNbO}_{3}$ (PPLN) crystal, pumped with up to $17 \mathrm{~W}$ of average power at $1064 \mathrm{~nm}$ in 16 -ps pulses $(59 \mathrm{MHz}$ repetition rate) from a passively mode-locked Nd:YAG laser (modified version of [2] with 2 laser heads). Feedback is provided through a $2 \mathrm{~m}$ long large mode area holey fiber [3]. In first experiments, we obtain up to $4.4 \mathrm{~W}$ of average signal power at $1485-1582 \mathrm{~nm}$. The pulse durations (FWHM) are around $10 \mathrm{ps}$. The average idler power is up to $1.9 \mathrm{~W}$. The operation is stable over hours.

\section{Summary}

We demonstrated a novel concept for synchronously pumped OPOs, which is based on feedback through a singlemode fiber in combination with high parametric gain, leading to multi-watt average signal powers both in the femtosecond and picosecond regime. The devices are unusually insensitive to intracavity losses and have a very compact setup. Even in the femtosecond domain, the cavity length adjustment is uncritical.

1. J. Aus der Au, G. J. Spühler, T. Südmeyer, R. Paschotta, R. Hövel, M. Moser, S. Erhard, M. Karszewski, A. Giesen, U. Keller, "16.2 W average power from a diode-pumped femtosecond Yb:YAG thin disk laser," Opt. Lett. 25, 859 (2000).

2. G. J. Spühler, T. Südmeyer, R. Paschotta, M. Moser, K. J. Weingarten, U. Keller, "Passively mode-locked high-power Nd:YAG lasers with multiple laser heads," Appl. Phys. B 71, 19 (2000).

3. N. G. R. Broderick, H. L. Offerhaus, D. J. Richardson, R. A. Sammut, "Power scaling in passively mode-locked large-mode area fiber lasers," IEEE Photonics Technol. Lett. 10, 1718 (1998). 